\title{
Network Based Approach in the Establishment of the Relationship between Type 2 Diabetes Mellitus and Its Complications at the Molecular Level Coupled with Molecular Docking Mechanism
}

\author{
Shailima Rampogu' and Mary Rampogu Lemuel ${ }^{2}$ \\ ${ }^{1}$ Celesta Research Lab, Hyderabad, Telangana 500 076, India \\ ${ }^{2}$ West Thames College, London TW7 4HS, UK \\ Correspondence should be addressed to Shailima Rampogu; shailima.rampogu@gmail.com
}

Received 7 June 2016; Accepted 11 July 2016

Academic Editor: Michele Migliore

Copyright (C) 2016 S. Rampogu and M. Rampogu Lemuel. This is an open access article distributed under the Creative Commons Attribution License, which permits unrestricted use, distribution, and reproduction in any medium, provided the original work is properly cited.

\begin{abstract}
Diabetes mellitus (DM) is one of the major metabolic disorders that is currently threatening the world. DM is seen associated with obesity and diabetic retinopathy (DR). In the present paper we tried to evaluate the relationship between the three aliments at the gene level and further performed the molecular docking to identify the common drug for all the three diseases. We have adopted several software programs such as Phenopedia, VennViewer, and CDOCKER to accomplish the objective. Our results revealed six genes that commonly associated and are involved in the signalling pathway. Furthermore, evaluation of common gene association from the selected set of genes projected the presence of SIRT1 in all the three aliments. Therefore, we targeted protein $4 \mathrm{KXQ}$ which was produced from the gene SIRT1 and challenged it with eight phytochemicals, adopting the CDOCKER. C1 compound has displayed highest -CDOCKER energy and -CDOCKER interaction energy of 43.6905 and 43.3953, respectively. Therefore, this compound is regarded as the most potential lead molecule.
\end{abstract}

\section{Introduction}

Globally, type 2 diabetes mellitus (T2DM) is one of the leading causes of death and it is estimated that over $70 \%$ of the population effected with T2DM are in the developing countries, China leading the world with 92.4 million $[1,2]$. Once a patient is diagnosed with T2DM, lento develops the other diseases. It is generally noted that the T2DM patients are obese; however, the molecular association that exists between them is far from clear. Statistical data show an alarming figure, with $34 \%$ of the US adults being obese [3] and it may soon increase by $21 \%$ in another three decades [4]. It is well evidenced that obese men are more prone to develop T2DM than obese women at the ratio of $11.2: 10$. Nevertheless, it has to be noted that several patients with unrestrained weight again are not likely to develop T2DM, which warrants the need for understanding their relationship at the molecular level [3]. Another complication, which is seen associated with
T2DM, is the diabetic retinopathy (DR) gradually causing visual impairment leading to blindness [5]. Recent reports evidently state the relationship between the BMI and DR [6]. Generally, BMI is considered as a determinant factor for obesity [7]. Wei et al. [8] also reported association between them at the genetic level. It is, hence, extremely essential to identify the relationship that exists between all the three diseases, majorly focusing on which disease is the initiator of the other two, and further to identify a common drug that could be a potential lead for all the diseases. To achieve this we have depended upon the system biology, which gives freedom in evaluating the biological system at the gene level. Additionally, this paper also makes an effort to identify the common genes and protein involved with the three aliments.

The objective of the present paper is to identify the genes representing the individual diseases and, further, the common genes involved and later to perform molecular docking to determine an effective drug molecule. 


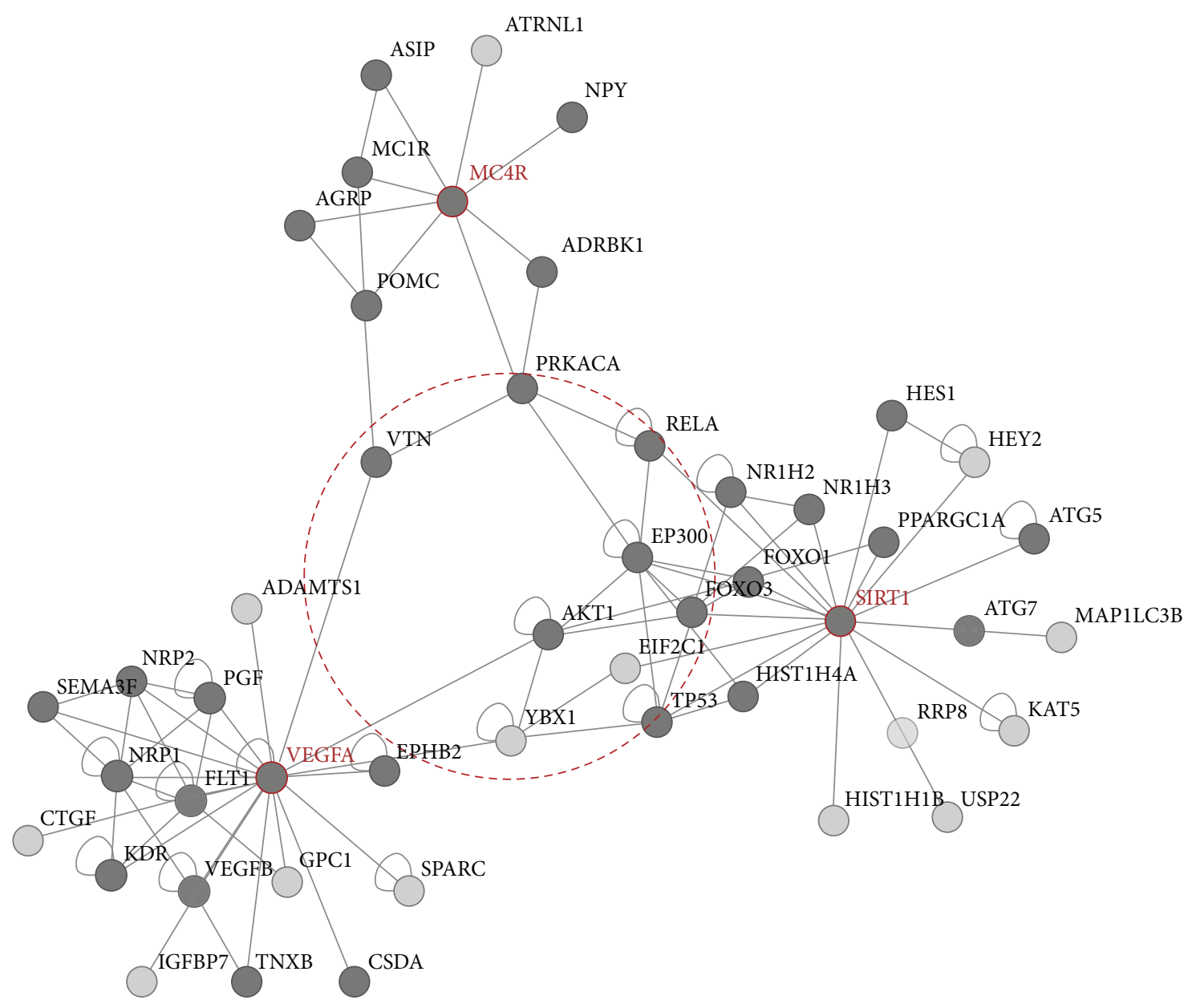

FIGURE 1: Red nodes indicate the query proteins and the proteins present in the dotted circles, VTN, PRKACA, RELA, EP300, AKT1, EIF2C1, are the common proteins associated with the query proteins. Dark grey nodes represent the signalling pathway members and the light grey nodes represent the nonsignalling pathway members.

\section{Materials and Methods}

2.1. Identification of the Common Genes. To identify the common genes, we have employed Phenopedia [9], Public Healthy Genomics Knowledge Base (V1.0), to search for the genes responsible of the diseases and, consequently, the genes responsible for the three aliments were identified. Later, they were imported onto the pathway linkers [10] to understand the significant common genes associated with all the three diseases, which are precisely involved in the signalling pathways.

Furthermore, in order to understand the disease-gene relationship, we relied on VennViewer, provided with the Comparative Toxicogenomics Databases, which has an ability to develop the Venn diagrams pertaining to three genes, diseases, or chemicals. For the present investigation, we worked with three diseases.

2.2. Protein Ligand Docking. In order to identify the candidate drug molecules, it is very essential to perform the protein ligand docking, modelling technique used to predict the orientation, and the position of the ligand upon docking.

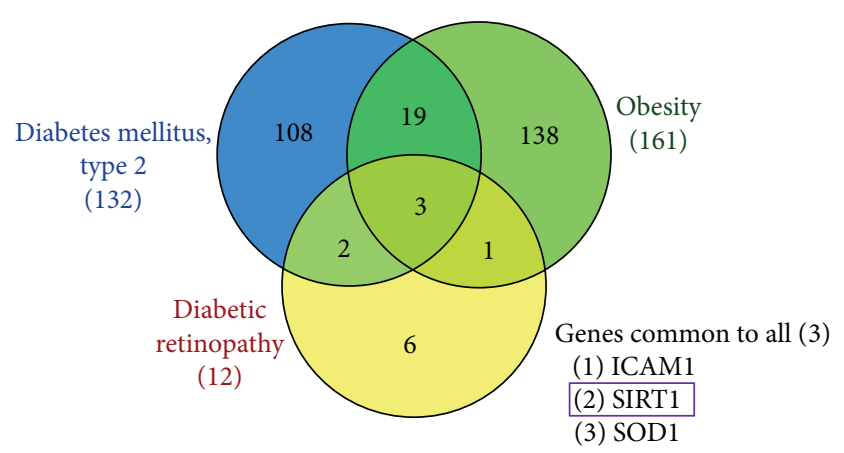

FIgURE 2: VennViewer diagram displaying the common genes associated with the three diseases.

For the present investigation, the CDOCKER, available on the Discovery Studio, was adopted. CDOCKER specifically employs the CHARMm-based molecular dynamics method and further generates conformation adopting the high temperature and is then forwarded onto the binding site for binding pose analysis. 
TABLE 1: Ligand name and structure.

Ligand name

C2<smiles>[Z]N1C(=O)C(O)C1C(O)C(O)[Z]([H])([H])[H]</smiles>

C3<smiles>O=c1c(O)c(-c2ccc(O)c(O)c2)oc2cc(O)cc(O)c12</smiles>

C4

$$
\text { (n) }
$$

C5

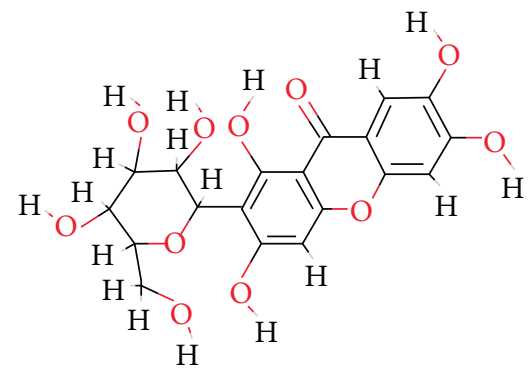

C6<smiles>O=C(O)c1cc(O)c(O)c(O)c1</smiles>

TABLE 1: Continued.
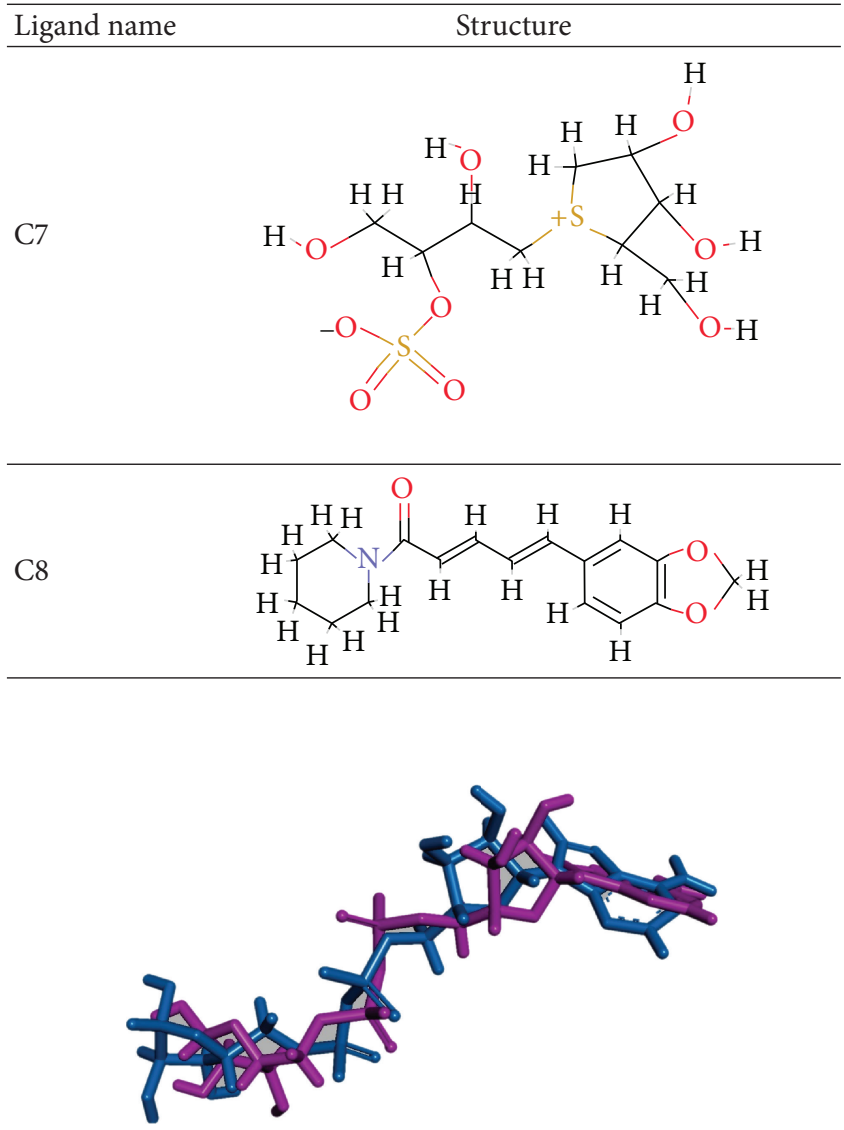

FIGURE 3: Docking of the cocrystal represented in magenta. Blue indicates the docked pose.

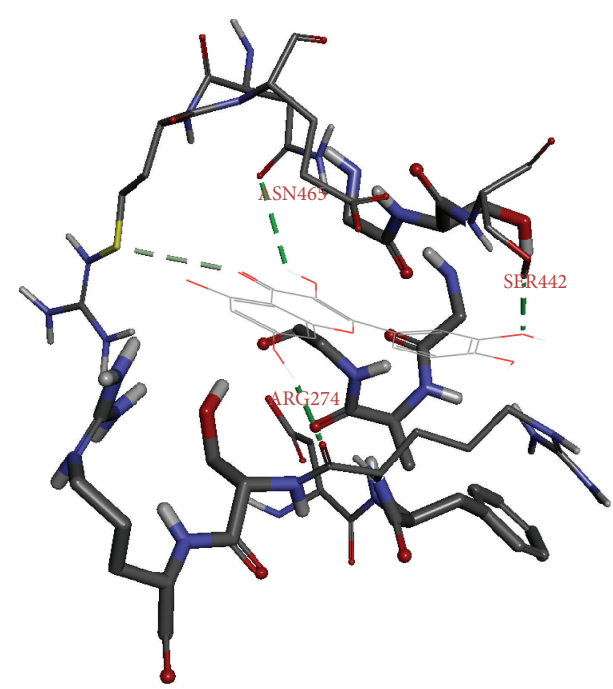

FIGURE 4: Protein ligand docking. Green dashed lines represent the hydrogen bond interactions.

2.3. Protein Selection and Preparation. The protein selection for the present investigation is one of the most crucial aspects. Since we aim at identifying the protein from the common gene, we relied upon $4 \mathrm{KXQ}$, a protein produced from the gene 


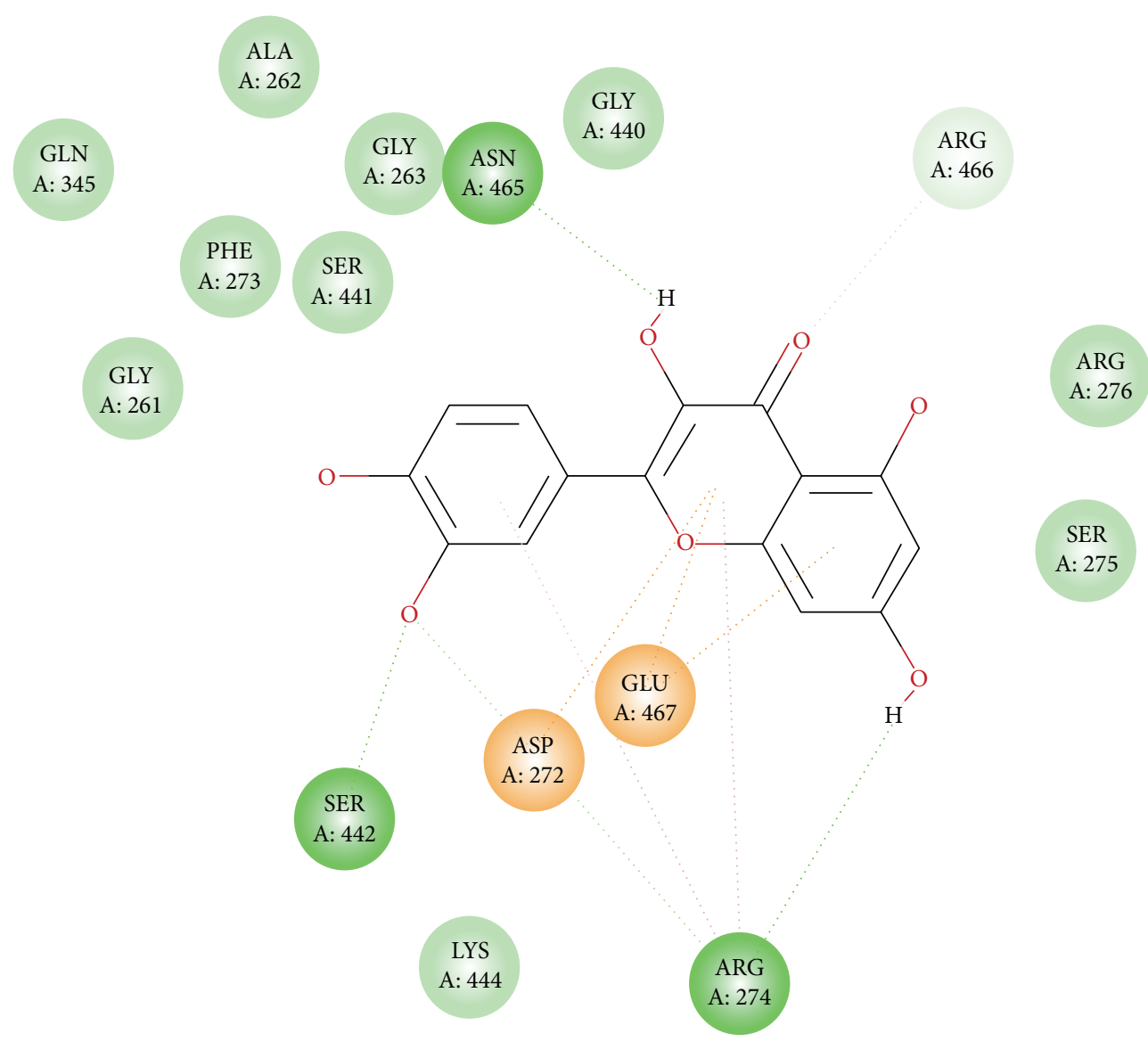

FIGURE 5: 2D representation of the protein ligand interaction. Brown dotted lines indicate the pi-anion bonds, pink dotted lines denote the pi-alkyl bonds, and the light green dotted lines represent the van der Waals interactions.

SIRT1 with the resolution of $1.85 \AA$ and is known as NADdependent protein deacetylase which envisages developing a common drug for all the three diseases.

The selected protein was prepared prior to the docking studies by correcting the chemistry of the missing hydrogens and the unfilled valence atoms. Thereafter, the protein was subjected to energy minimization by applying the CHARMm force field until a satisfactory gradient tolerance was obtained.

2.4. Ligand Preparation. A total of eight natural compounds were chosen to challenge against the protein target molecule. These compounds were drawn on Marvin Sketch and their corresponding 3D structures were generated on the DS. CHARMm force field was applied as a measure to minimize the ligand molecules. The importance of choosing the natural compounds is to further formulate and translate them into nutraceuticals, Table 1.

\section{Results and Discussions}

3.1. Identification of the Common Genes. Phenopedia was employed to identify the genes associated with obesity, diabetes mellitus, and diabetic retinopathy, respectively. A systematic search was conducted providing the disease names as a query. Consequently, SIRT1, MC4R, and VEGFA were determined for diabetes mellitus, type 2, obesity, and diabetic retinopathy, respectively.

Later, they were assessed for the common genes on pathway linkers that have an ability to link proteins to the signalling pathways. A total of 48 proteins were found to be associated; nevertheless, only six proteins were seen interacting with all the three genes. In addition, all the six proteins were involved in the signalling pathways and the nonsignalling proteins were ignored, Figure 1.

Alternatively, we tried to evaluate the gene that is involved in all the three disorders and in this pursuit, we adopted the VennViewer [11] available on Comparative Toxicogenomics Database (CTD) facilitating the three genes of interest as inputs. Following which, the results were generated pronouncing SIRT1 to be involved with all the three diseases, Figure 2. Additionally, we have identified two other genes, ICAM1 and SOD1, that were seen involved with the three diseases.

3.2. Active Site Identification. Active site was identified based upon the cocrystal and all the amino acids around the cocrystals were taken into consideration. Furthermore, the cocrystal docking was performed to ascertain the active site and an 
TABLE 2: CDOCKER scores.

\begin{tabular}{|c|c|c|}
\hline Compound name & -CDOCKER energy & -CDOCKER interaction \\
\hline $\mathrm{Cl}$ & 43.6905 & 46.3953 \\
\hline $\mathrm{C} 2$ & 43.594 & 46.2159 \\
\hline $\mathrm{C} 3$ & 43.4827 & 46.2751 \\
\hline $\mathrm{C} 4$ & 41.0953 & 43.8479 \\
\hline C5 & 39.5149 & 45.9837 \\
\hline C6 & 38.0443 & 41.3656 \\
\hline $\mathrm{C} 7$ & 37.9135 & 41.3597 \\
\hline $\mathrm{C} 8$ & 37.8987 & 41.12 \\
\hline $\mathrm{C} 9$ & 37.8849 & 41.0074 \\
\hline $\mathrm{C} 10$ & 36.7593 & 44.0819 \\
\hline C11 & 34.1467 & 30.8196 \\
\hline $\mathrm{C} 12$ & 34.0604 & 30.898 \\
\hline $\mathrm{C} 13$ & 33.9507 & 30.5325 \\
\hline $\mathrm{C} 14$ & 33.8314 & 30.5452 \\
\hline $\mathrm{C} 15$ & 33.7333 & 30.2313 \\
\hline $\mathrm{C} 16$ & 33.6998 & 30.4529 \\
\hline $\mathrm{C} 17$ & 33.6256 & 30.2404 \\
\hline $\mathrm{C} 18$ & 33.3807 & 29.9087 \\
\hline $\mathrm{C} 19$ & 33.3558 & 29.9264 \\
\hline $\mathrm{C} 20$ & 33.3249 & 29.8632 \\
\hline $\mathrm{C} 21$ & 16.1116 & 43.6807 \\
\hline $\mathrm{C} 22$ & 15.345 & 45.089 \\
\hline $\mathrm{C} 23$ & 13.6468 & 43.0185 \\
\hline $\mathrm{C} 24$ & 11.3323 & 33.3176 \\
\hline $\mathrm{C} 25$ & 11.117 & 29.8403 \\
\hline $\mathrm{C} 26$ & 10.8676 & 39.7459 \\
\hline $\mathrm{C} 27$ & 10.6943 & 36.1858 \\
\hline $\mathrm{C} 28$ & 6.76306 & 39.212 \\
\hline $\mathrm{C} 29$ & 6.74011 & 29.1499 \\
\hline $\mathrm{C} 30$ & 6.5533 & 28.9768 \\
\hline $\mathrm{C} 31$ & 6.37705 & 26.9035 \\
\hline $\mathrm{C} 32$ & 6.2874 & 27.1806 \\
\hline C33 & 6.10317 & 27.0072 \\
\hline C34 & 5.97667 & 26.6726 \\
\hline C35 & 5.92624 & 27.6206 \\
\hline C36 & 5.92438 & 26.961 \\
\hline C37 & 5.85956 & 26.796 \\
\hline C38 & 5.85111 & 26.9232 \\
\hline C39 & 5.82535 & 30.565 \\
\hline $\mathrm{C} 40$ & 5.72081 & 26.7008 \\
\hline C41 & 5.42032 & 42.1491 \\
\hline $\mathrm{C} 42$ & 5.2531 & 32.1618 \\
\hline $\mathrm{C} 43$ & 4.22422 & 40.4729 \\
\hline $\mathrm{C} 44$ & 3.15406 & 40.3832 \\
\hline C45 & 2.38138 & 36.5095 \\
\hline $\mathrm{C} 46$ & 2.31672 & 38.5722 \\
\hline $\mathrm{C} 47$ & 2.27477 & 36.9574 \\
\hline $\mathrm{C} 48$ & 1.9534 & 36.4364 \\
\hline $\mathrm{C} 49$ & 1.49815 & 36.2616 \\
\hline C50 & 1.18856 & 38.0515 \\
\hline
\end{tabular}

TABLE 2: Continued.

\begin{tabular}{lcc}
\hline Compound name & -CDOCKER energy & -CDOCKER interaction \\
\hline C51 & 0.420181 & 22.2711 \\
C52 & 0.183442 & 23.049 \\
C53 & 0.0514005 & 48.6528 \\
C54 & -1.0173 & 26.8177 \\
C55 & -1.88654 & 45.5105 \\
C56 & -2.08119 & 47.3119 \\
C57 & -2.33309 & 48.7866 \\
C58 & -2.40939 & 41.621 \\
C59 & -3.58299 & 42.0705 \\
C60 & -4.44257 & 41.6539 \\
C61 & -4.72479 & 41.9058 \\
C62 & -4.92736 & 45.634 \\
C63 & -5.16567 & 44.7624 \\
C64 & -6.0176 & 39.6754 \\
C65 & -6.11149 & 39.7136 \\
C66 & -7.27294 & 42.9186 \\
C67 & -13.8456 & 32.8447 \\
C68 & -16.8805 & 32.324 \\
C69 & -19.8347 & 37.0598 \\
C70 & -19.8904 & 32.4273 \\
\hline
\end{tabular}

acceptable RMSD of 0.9 was obtained projecting that our docking parameters are valid ones, Figure 3.

3.3. Molecular Docking Mechanism. Molecular docking was performed adopting the CDOCKER, which depends on CHARMm-based force field. Subsequently, diverse poses are generated adopting the random rigid body rotation and simulate annealing. In order to initiate this mechanism, all the default parameters were considered allowing the generation of 10 poses for every ligand. The docking estimation was performed by the -CDOCKER energy, which was calculated, based upon the internal ligand strain energy and receptorligand interaction energy. Additionally, -CDOCKER interaction signifies the energy of the nonbonded interaction that exists between the protein and the ligand. In both the cases, it has to be noted that greater -CDOCKER energy and -CDOCKER interaction energy value implies greater favourable binding between the protein and the ligand.

As mentioned above, eight naturally available phytochemicals were challenged with the protein target. Seven ligands have displayed an efficient docking with the generation of 10 conformers each; however, one ligand failed to dock. The representative dock results are displayed in Table 2. Among the docked ligands, $\mathrm{Cl}$ displayed higher -CDOCKER energy and -CDOCKER interaction energy value-making itself the potential leading molecule for the three common diseases. Furthermore, the protein ligand complex was assessed for the hydrogen bond interaction followed by the binding mode analysis. Delineating on the interactions reveals that the residues, ASN465, SER442, and ARG274, have participated in the hydrogen bond formation, Figure 4, while the amino 
acid ARG466 participated by Vander Walls interaction and ASP272 and GLU467 interacted by the Pi-anion and ARG274 with Pi-alkyl bonds, respectively, Figure 5. Regardless of the binding energies projected by the ligands, they all obeyed the same pattern of binding mode as with the cocrystal.

\section{Discussion}

It has been a long subject of debate regarding the diabetes mellitus type 2 and its associated complication [12]. However, nothing concrete has yet been established. In the present paper, we have successfully evaluated the common genes associated with the three diseases. These findings could lead the researchers towards unfolding the mystery behind the diabetes complications. In the event of identifying the common genes associated among the three selected genes, our results determine SIRT1 to be the link gene, a gene which was chosen for diabetes mellitus. Therefore, it can be deduced that diabetes mellitus can influence the manifestation of obesity and diabetic retinopathy. Furthermore, our study centralizes on the identification of a common nutraceutical for all the three aliments. Accordingly, we have preferred the protein $4 \mathrm{KXQ}$, a protein produced from the gene SIRT1. We have further evidenced the presence of SIRT1 in DM, obesity, and DR [13-15]. Following this, we challenged the selected protein with eight natural compounds or the phytochemicals. Natural compounds offer a host of applications such as low cost, wider availability, and low side effects which are a few to mention. Additionally, they can be supplemented through diet. Amongst all the ligand molecules, $\mathrm{Cl}$ emerged as the best ligand demonstrating a highest -CDOCKER interaction value of 46.3953 and its corresponding -CDOCKER energy of 43.6905 , respectively.

In summary, our results signify being of greater scientific usefulness in finding the most prominent results in combating the diabetes complications.

\section{Competing Interests}

The authors declare that they have no competing interests.

\section{References}

[1] L. B. Rawal, R. J. Tapp, E. D. Williams, C. Chan, S. Yasin, and B. Oldenburg, "Prevention of type 2 diabetes and its complications in developing countries: a review," International Journal of Behavioral Medicine, vol. 19, no. 2, pp. 121-133, 2012.

[2] W. Yang, J. Lu, J. Weng et al., "Prevalence of diabetes among men and women in China," The New England Journal of Medicine, vol. 362, no. 12, pp. 1090-1101, 2010.

[3] R. H. Eckel, S. E. Kahn, E. Ferrannini et al., "Obesity and type 2 diabetes: what can be unified and what needs to be individualized?” Diabetes Care, vol. 34, no. 6, pp. 1424-1430, 2011.

[4] J. P. Boyle, T. J. Thompson, E. W. Gregg, L. E. Barker, and D. F. Williamson, "Projection of the year 2050 burden of diabetes in the US adult population: dynamic modeling of incidence, mortality, and prediabetes prevalence," Population Health Metrics, vol. 8, no. 1, article 29, 2010.
[5] V. Swetha and E. Jeganathan, "Ocular associations of diabetes other than diabetic retinopathy," Diabetes Care, vol. 31, no. 9, pp. 1905-1912, 2008.

[6] S. Kaštelan, M. Tomić, A. Gverović Antunica, S. Ljubić, J. Salopek Rabatić, and M. Karabatić, "Body mass index: a risk factor for retinopathy in type 2 diabetic patients," Mediators of Inflammation, vol. 2013, Article ID 436329, 8 pages, 2013.

[7] R. Raman, P. K. Rani, P. Gnanamoorthy, R. R. Sudhir, G. Kumaramanikavel, and T. Sharma, "Association of obesity with diabetic retinopathy: Sankara Nethralaya Diabetic Retinopathy Epidemiology and Molecular Genetics study (SN-DREAMS Report no. 8)," Acta Diabetologica, vol. 47, no. 3, pp. 209-215, 2010.

[8] F. Wei, C. Cai, S. Feng et al., "TOX and CDKN2A/B gene polymorphisms are associated with type 2 diabetes in Han Chinese," Scientific Reports, vol. 5, Article ID 11900, 2015.

[9] W. Yu, M. Clyne, M. J. Khoury, and M. Gwinn, "Phenopedia and Genopedia: disease-centered and gene-centered views of the evolving knowledge of human genetic associations," Bioinformatics, vol. 26, no. 1, pp. 145-146, 2010.

[10] I. J. Farkas, Á. Szántó-Várnagy, and T. Korcsmáros, "Linking proteins to signaling pathways for experiment design and evaluation," PLoS ONE, vol. 7, no. 4, Article ID e36202, 2012.

[11] C. J. Grondin, A. P. Davis, T. C. Wiegers et al., "Advancing exposure science through chemical data curation and integration in the comparative toxicogenomics database," Environmental Health Perspectives, 2016.

[12] J. Rosenstock, "Reflecting on type 2 diabetes prevention: more questions than answers!," Diabetes, Obesity and Metabolism, vol. 9, no. 1, pp. 3-11, 2007.

[13] M. Kitada and D. Koya, "SIRT1 in type 2 diabetes: mechanisms and therapeutic potential," Diabetes and Metabolism Journal, vol. 37, no. 5, pp. 315-325, 2013.

[14] M. C. Zillikens, J. B. J. Van Meurs, F. Rivadeneira et al., "SIRT1 genetic variation is related to BMI and risk of obesity," Diabetes, vol. 58, no. 12, pp. 2828-2834, 2009.

[15] S. Liu, Y. U. Lin, and X. Liu, "Protective effects of SIRT1 in patients with proliferative diabetic retinopathy via the inhibition of IL-17 expression," Experimental and Therapeutic Medicine, vol. 11, no. 1, pp. 257-262, 2016. 


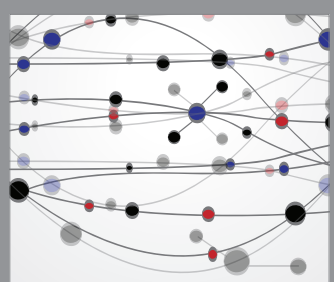

The Scientific World Journal
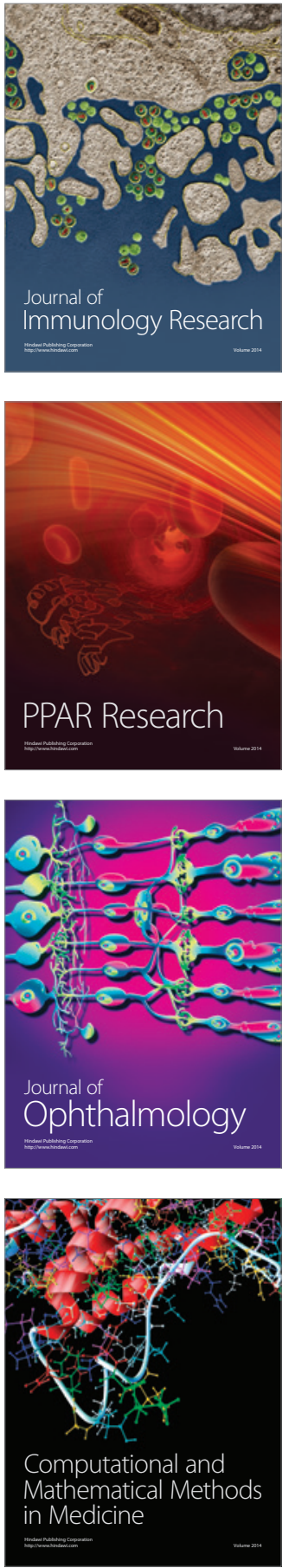

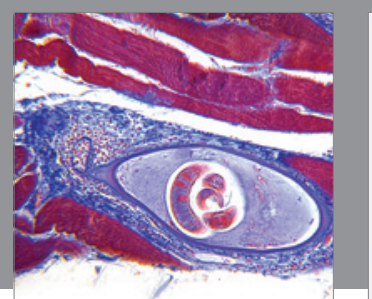

Gastroenterology Research and Practice

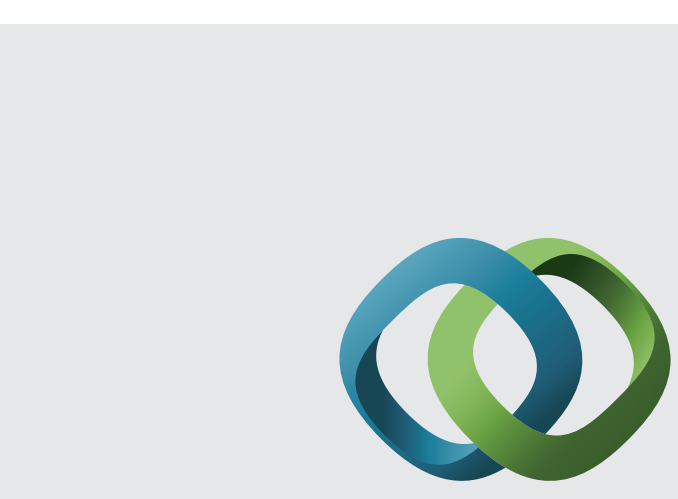

\section{Hindawi}

Submit your manuscripts at

http://www.hindawi.com
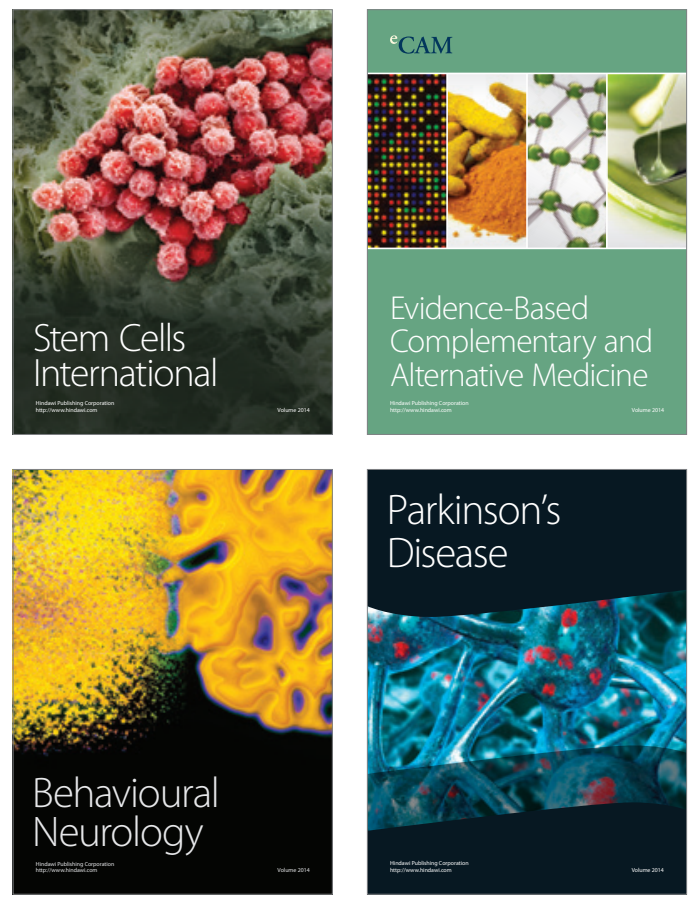
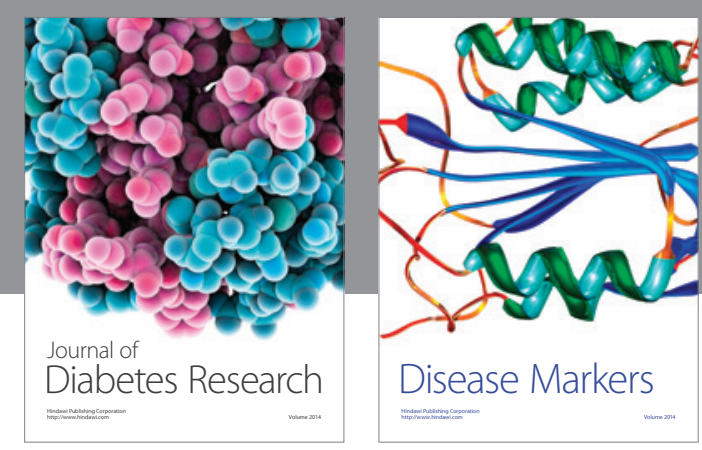

Disease Markers
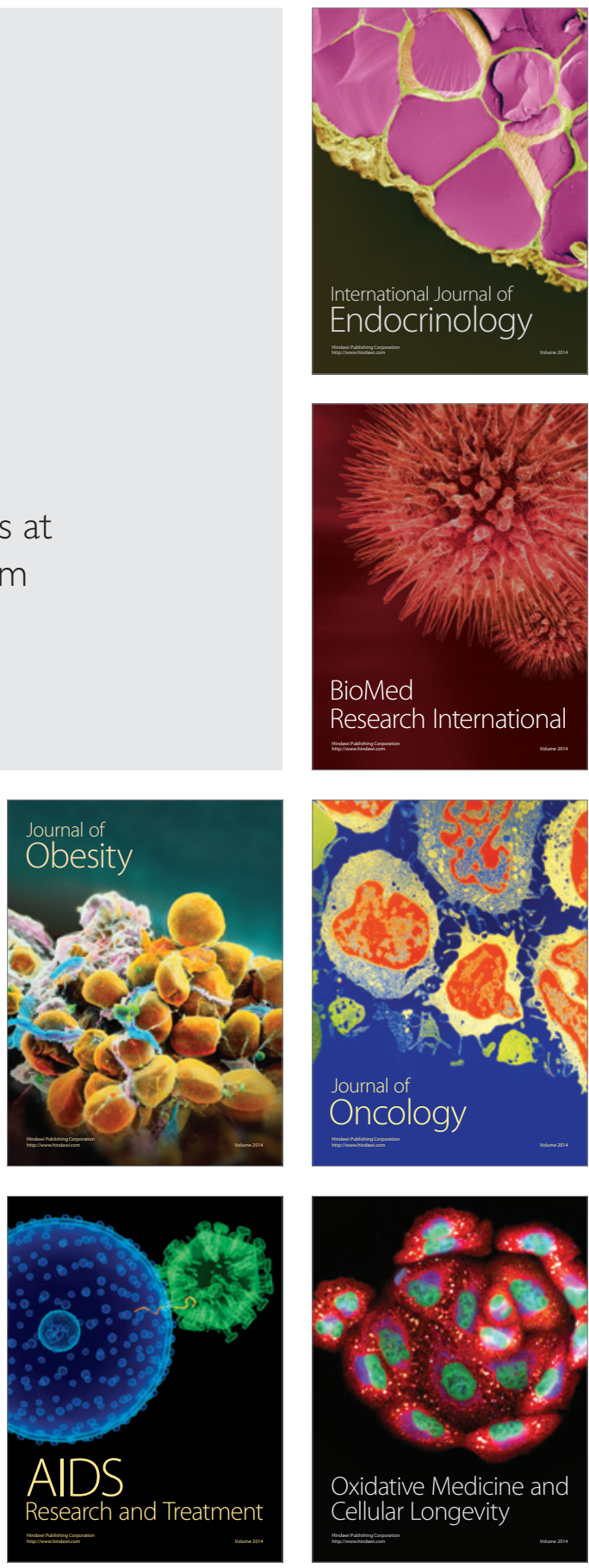